\title{
SEDUM MOCINIANUM (CRASSULACEAE) UNA ESPECIE NUEVA DEL CENTRO DE MEXICO ${ }^{1}$
}

\author{
Emmanuel Perez-Calix \\ Instituto de Ecología, A.C. \\ Centro Regional del Bajío \\ Apartado postal 386 \\ 61600 Pátzcuaro, Michoacán
}

\begin{abstract}
RESUMEN
Se describe y propone como especie nueva para la ciencia a Sedum mocinianum. El nuevo taxon se ubica en la sección Sedastrum (Rose) Berger, por presentar hojas dispuestas en una roseta basal, inflorescencia en panícula, corola de color blanco, una concavidad en la base de los carpelos, así como pubescencia en diferentes órganos. La nueva especie se relaciona estrechamente con Sedum hintonii R. T. Clausen, del cual difiere en su inflorescencia más corta y estrecha, así como en las hojas del talllo floral, que son elípticas a oblongas.
\end{abstract}

\section{ABSTRACT}

Sedum mocinianum is described, and proposed as a new species. This taxon belongs to the section Sedastrum (Rose) Berger, on account of its leaves arranged in a basal rosette, paniculate inflorescence, white corolla, carpels concave under the scales, as well as the pubescense of several organs. The new species is related to Sedum hintonii R. T. Clausen from which it can be distinguished by its narrow and short inflorescence, as well as by the leaves of the inflorescence, which in this species are elliptic to oblong.

En una exploración realizada a la Sierra de Los Agustinos, en el municipio de Acámbaro, Guanajuato, se colectó una planta del género Sedum (Crassulaceae), la que al intentar determinar se identificó inicialmente como Sedum hintonii R. T. Clausen. Sin embargo, al compararla con la descripción y dibujo originales de tal especie (Clausen, 1943), se observó que difiere de ella y de otras afines en la morfología de la hoja y de la inflorescencia, por lo que se concluyó que es una entidad diferente de las ya conocidas.

Clausen diferenció a $S$. hintonii de las demás especies de la sección Sedastrum en lo abundante y peculiar de la pubescencia, así como en las hojas caulinas que son pequeñas y lanceoladas. Sin embargo, en las claves lo separa basándonse únicamente en la densidad de la pubescencia (Clausen, 1943, 1984) lo que, en parte, ha ocasionado que se identifiquen incorrectamente ejemplares con este nombre, como es el caso de Jacobsen (1960) y

1 Trabajo realizado con apoyo económico del Instituto de Ecología, A.C. (cuenta 902-03), del Consejo Nacional de Ciencia y Tecnología y de la Comisión Nacional para el Conocimiento y Uso de la Biodiversidad. 
Stephenson (1994), quienes publicaron fotos y pequeñas diagnosis de plantas que reconocieron como Sedum hintonii. Tales plantas más bien son similares a la que se describe aquí.

Sedum mocinianum E. Pérez-Calix sp. nov. Fig. 1.

Planta herbacea perennis, nunc caulibus pendulis ramosis usque $80 \mathrm{~cm}$ longis, nunc dense rosulata; caules, folia, pedunculi, inflorescentiae rami, bracteae, sepalaque dense pubescentes pilis hyalinis ca. $0.5 \mathrm{~mm}$ longis; folia in rosulis densis disposita vel spiraliter ad caulis apicem ordinata, elliptica usque oblongo-elliptica, $0.8-2.5 \mathrm{~cm}$ longa, $0.6-1.1 \mathrm{~cm}$ lata, $0.5 \mathrm{~cm}$ crassa, in apice obtusa; inflorescentia thyrsiformis, pedunculo $2-3.5 \mathrm{~cm}$ longo, bracteis $0.4-0.9 \mathrm{~cm}$ longis, $0.3-0.7 \mathrm{~cm}$ latis; flores sessiles vel subsessiles; calycis lobi 5 , ad basem connati subaequales oblongi, 2.5-3.0 mm longi, 1.5-2.0 mm lati; petala alba; nectaria anguste oblonga vel ovata; carpella ad basem exarata; folliculi seminibus plurimis.

Planta herbácea perenne, tallos colgantes de hasta $80 \mathrm{~cm}$ de largo y $0.5 \mathrm{~cm}$ de diámetro, ramificados en ramitas arrosetadas, o bien, plantas con tallos muy cortos formando manchones densos a manera de cojines; tallos, hojas, pedúnculo, brácteas, ramas de la inflorescencia y sépalos densamente pubescentes, pelos hialinos de $0.5 \mathrm{~mm}$ de largo; hojas dispuestas en rosetas densas o en espiral en la porción superior del tallo, elípticas a oblongo-elípticas, de 0.8 a $2.5 \mathrm{~cm}$ de largo por 0.6 a $1.1 \mathrm{~cm}$ de ancho y $\pm 0.5 \mathrm{~cm}$ de grueso, ápice obtuso; inflorescencia tirsoidea, con hasta 10 ramificaciones en cincino con 1 a 3 flores en cada rama; pedúnculo de 2 a $3.5 \mathrm{~cm}$ de alto y $0.4 \mathrm{~cm}$ de diámetro; brácteas dispuestas en espiral, similares en forma a las hojas de la roseta, de 0.4 a $0.9 \mathrm{~cm}$ de largo por 0.3 a $0.7 \mathrm{~cm}$ de ancho y 0.2 a 0.5 de grueso; flores sésiles o subsésiles; cáliz de 5 lóbulos, fusionados en la base formando un tubo de $\pm 1 \mathrm{~mm}$ de alto, lóbulos subiguales, oblongos, de 2.5 a $3.0 \mathrm{~mm}$ de largo por 1.5 a $2.0 \mathrm{~mm}$ de ancho en la base, ápice obtuso; pétalos 5, libres, de color blanco, oblongo-lanceolados, extendidos en la parte media, de $5 \mathrm{~mm}$ de largo por $3 \mathrm{~mm}$ de ancho en la región más amplia; estambres 10, 5 opuestos y adnados a los pétalos, de $2 \mathrm{~mm}$ de largo, 5 alternos de $4 \mathrm{~mm}$ de largo; nectarios estrechamente oblongos u ovados, de $\pm 0.4 \mathrm{~mm}$ de largo, carpelos de $5 \mathrm{~mm}$ de largo con una concavidad en la base, de color verde tierno, estilos de $1.5 \mathrm{~mm}$ de largo, filamentosos; folículos con numerosas semillas.

TIPO: México, Guanajuato, San Luis de Los Agustinos, municipio de Acámbaro. Alt. 2,400 m. Bosque tropical caducifolio. Crece en las grietas de las paredes de rocas ígneas, particularmente en lugares sombreados, 29.I.1998, E. Pérez, C. Glass y M. Mendoza 3797 (IEB).

Material adicional examinado: Guanajuato, San Luis de Los Agustinos, municipio de Acámbaro, E. Pérez y C. Glass 3592 (IEB).

Sedum mocinianum se conoce con exactitud solamente de la localidad de donde proviene el tipo, aunque es probable que su distribución sea más amplia, dadas las referencias publicadas en las que se ha identificado erróneamente como Sedum hintonii. 
Pérez-Calix: Sedum mocinianum (Crassulaceae) Una Nueva Especie del Centro de México

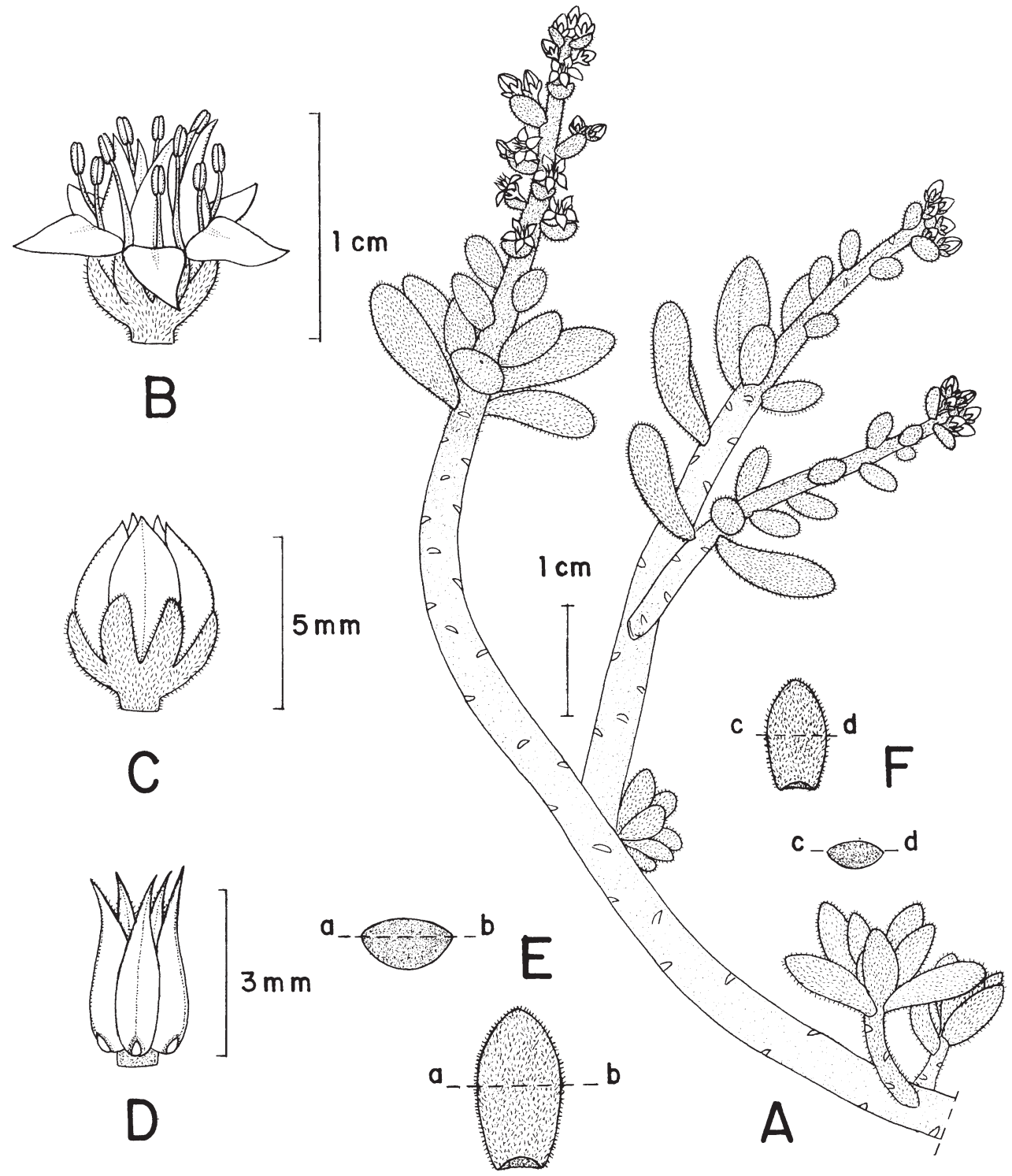

Fig. 1. Sedum mocinianum Pérez-Calix. A. Hábito de la planta, B. Flor en vista lateral, C. Botón floral, D. Carpelos y nectarios, E. Hoja en vista ventral, a-b, sección transversal de la hoja, F. Bráctea en vista ventral, c-d, sección transversal de la bráctea. (Dibujo realizado por el Sr. Rogelio Cárdenas Soriano, basado en E. Pérez, C. Glass y M. Mendoza 3797 (IEB)). 
Así, Clausen (1981) menciona que la planta que el vio es originaria de una localidad denominada Cañón del Río Ventana, en el estado de Durango, mientras que el material observado por Stephenson (1994), es de origen cultivado de procedencia desconocida, probablemente de Las Canoas en el mismo estado de Durango.

Uhl (1992) contó los cromosomas de plantas determinadas como Sedum hintonii, pero probablemente el material que revisó para su conteo pertenece a Sedum mocinianum, de manera que el número cromosómico $(n=25)$ observado por Uhl correspondería a esta última especie.

Por ser una planta herbácea perenne, durante la mayor parte del año se puede localizar en estado vegetativo, en floración se le ha encontrado sólo de finales de enero a principios de marzo.

El nuevo taxon se ubica en la sección Sedastrum (Rose) Berger por sus hojas basales que forman rosetas densas, sus corolas de color blanco, su inflorescencia más o menos paniculada, así como por los carpelos erectos con una concavidad en la base, en la que se inserta la escama nectarífera. La sección Sedastrum, como se conoce en la actualidad, está integrada por Sedum ebracteatum DC., S. glabrum (Rose) Praeger, S. hemsleyanum Rose y $S$. hintonii R. T. Clausen. De todas ellas Sedum mocinianum se diferencia en la inflorescencia más corta y estrecha, ya que en las demás especies la panícula es laxa y con ramificaciones largas; de Sedum ebracteatum, S. glabrum y de $S$. hemsleyanum se separa además por la densidad de la pubescencia.

Aparentemente Sedum hintonii es el taxon más afín, pero la panícula tan estrecha (tirso) de Sedum mocinianum en comparación con la inflorescencia laxa y de mayores dimensiones de $S$. hintonii, al igual que las hojas del tallo floral elípticas a oblongas de S. mocinianum, no lanceoladas como en $S$. hintonii, diferencian a ambas especies (Cuadro 1).

Cuadro 1. Comparación de algunos caracteres de Sedum mocinianum con los de las otras especies de la Sección Sedastrum.

\begin{tabular}{|c|c|c|c|c|c|}
\hline & S. mocinianum & S. hintonii & S. hemsleyanum & S. ebracteatum & S. glabrum \\
\hline \multicolumn{6}{|l|}{ Hojas } \\
\hline forma & $\begin{array}{l}\text { elíptica a } \\
\text { oblongo- } \\
\text { elíptica }\end{array}$ & $\begin{array}{l}\text { oblonga a } \\
\text { elíptica }\end{array}$ & $\begin{array}{c}\text { espatulada, } \\
\text { elíptica u } \\
\text { oblanceolada }\end{array}$ & $\begin{array}{c}\text { suborbicular a } \\
\text { oblongo- } \\
\text { espatulada }\end{array}$ & $\begin{array}{l}\text { oblonga a } \\
\text { oblongo- } \\
\text { espatulada }\end{array}$ \\
\hline largo $(\mathrm{cm})$ & $0.8-2.5$ & $1.5-5$ & $0.5-1$ & $1-3$ & $0.5-4.0$ \\
\hline ancho $(\mathrm{cm})$ & $0.6-1.1$ & $0.3-1.0$ & $0.1-0.3$ & $0.6-1.5$ & $0.2-1.8$ \\
\hline pubescencia & $\begin{array}{l}\text { densamente } \\
\text { pubescente }\end{array}$ & $\begin{array}{l}\text { densamente } \\
\text { pubescente }\end{array}$ & pubescente & pubescente & $\begin{array}{c}\text { glabra a } \\
\text { puberulenta }\end{array}$ \\
\hline \multicolumn{6}{|l|}{ Pedúnculo } \\
\hline largo $(\mathrm{cm})$ & $2-3.5$ & 24 & $10-30$ & $\begin{array}{l}\text { variable, pero } \\
\text { mayor de } 10\end{array}$ & $15-25$ \\
\hline pubescencia & $\begin{array}{l}\text { densamente } \\
\text { pubescente }\end{array}$ & $\begin{array}{l}\text { densamente } \\
\text { pubescente }\end{array}$ & $\begin{array}{l}\text { glabro o } \\
\text { pubescente }\end{array}$ & puberulento & glabro \\
\hline
\end{tabular}


Pérez-Calix: Sedum mocinianum (Crassulaceae) Una Nueva Especie del Centro de México

Cuadro 1. Continuación.

\begin{tabular}{|c|c|c|c|c|c|}
\hline & S. mocinianum & S. hintonii & S. hemsleyanum & S. ebracteatum & S. glabrum \\
\hline $\begin{array}{l}\text { Brácteas } \\
\text { forma }\end{array}$ & $\begin{array}{l}\text { elíptica a } \\
\text { oblongo- } \\
\text { elíptica }\end{array}$ & lanceolada & $\begin{array}{c}\text { linear, } \\
\text { elíptica u } \\
\text { oblanceolada }\end{array}$ & $\begin{array}{l}\text { ovada a } \\
\text { oblonga }\end{array}$ & oblonga \\
\hline largo $(\mathrm{cm})$ & $0.4-0.9$ & $1-2.5$ & $0.3-2.0$ & $2-8$ & $0.2-2.7$ \\
\hline ancho $(\mathrm{cm})$ & $0.3-0.7$ & $4-5$ & $0.5-.45$ & $1-2.5$ & $0.1-0.9$ \\
\hline $\begin{array}{l}\text { Infllorescencia } \\
\text { tipo }\end{array}$ & tirso & $\begin{array}{c}\text { panícula } \\
\text { abierta }\end{array}$ & panícula & panícula & panícula \\
\hline $\begin{array}{l}\text { Distribución } \\
\text { geográfica } \\
\text { conocida }\end{array}$ & $\begin{array}{c}\text { Gto. } \\
\text { (Eje Neovol- } \\
\text { cánico Trans- } \\
\text { versal) }\end{array}$ & $\begin{array}{c}\text { Mich. } \\
\text { (Sierra Madre } \\
\text { del Sur) }\end{array}$ & $\begin{array}{l}\text { Hgo., Mor., Pue., } \\
\text { Gro., Oax. } \\
\text { (Eje Neovolcánico } \\
\text { Transversal y } \\
\text { Sierra Madre del } \\
\text { Sur) }\end{array}$ & $\begin{array}{l}\text { Tamps., Dgo., } \\
\text { Zac., S.L.P., } \\
\text { Gto., Qro., Hgo., } \\
\text { Jal., Mich., Méx., } \\
\text { D.F., Pue., Gro., } \\
\text { Oax., Centro } \\
\text { América }\end{array}$ & $\begin{array}{c}\text { Coah., S.L.P., } \\
\text { (norte de la } \\
\text { Altiplanicie } \\
\text { Mexicana) }\end{array}$ \\
\hline
\end{tabular}

El epíteto específico del nuevo taxon se dedica como homenaje a José Mariano Mociño (1757-1820), integrante de La Real Expedición Botánica y quien, de acuerdo con Rzedowski (1992): "fue el primer botánico mexicano de la era moderna e indudablemente uno de los más grandes del país".

\section{AGRADECIMIENTOS}

Agradezco al Dr. Jerzy Rzedowski la lectura crítica del manuscrito, así como la traducción de la diagnosis al latín; a la maestra Graciela Calderón de Rzedowski y a la Biól. Rosa María Murillo Martínez las sugerencias y observaciones realizadas al manuscrito. Al Sr. Charles Glass y al Prof. Mario Mendoza su amable compañía en el trabajo de campo, particularmente durante la colecta del ejemplar tipo.

\section{LITERATURA CITADA}

Clausen, R. T. 1943. The section Sedastrum of Sedum. Bull. Torr. Bot. Club 70: 289-296.

Clausen, R. T. 1981. Variation of species of Sedum of the Mexican Cordilleran Plateau. Privately published by R. T. Clausen. Ithaca, N.Y. 27 pp. 
Clausen, R. T. 1984. Sedum (Crassulaceae) of the Mexican Cordilleran Plateau. Gentes Herb. 12: 848.

Jacobsen, H. 1960. A handbook of succulent plants. Blandford Press. Londres. Vol.2. pp. 781-782. Rzedowski, J. 1992. Presentación. In: Guevara, S., P. Moreno-Casasola y J. Rzedowski (compiladores). Logros y perspectivas del conocimiento de los recursos vegetales de México en vísperas del siglo XXI. Instituto de Ecología, A.C.-Sociedad Botánica de México, A.C. Xalapa, Veracruz. $230 \mathrm{pp}$.

Stephenson, R. 1994. Sedum, cultivated stonecrops. Timber Press Inc. Portland, Oregon. 335 pp. Uhl, C. H. 1992. Chromosomes of Mexican Sedum VI. Section Sedastrum. Rhodora 94: 362-370.

Aceptado para publicación en agosto de 1998. 